sulphur-containing amino-acids such as cysteine and methionine, probably as a result of excessive cyanide detoxication, and the plasma thiocyanate was raised. This work, in conjunction with that of A. D. MacKenzie and C. I. Phillips ${ }^{12}$ on West Indian amblyopia, suggests that the dietary ingestion of cyanide is probably the most important single aetiological factor, though associated deficiencies of certain vitamins of the B group may possibly play a part also in causing this type of tropical neuropathy. The hope of preventing the syndrome would seem to depend on finding some means of reducing the dietary intake of cyanide and of providing the population at risk with a more balanced diet.

\section{Teaching Medicine to Dental Students}

Dentistry is the only specialty of surgery which can be studied in some depth as an undergraduate and for the practice of which a basic medical qualification is not required. Even though the dental student does not undertake a full undergraduate medical training an adequate background of general medical knowledge must be given to him. Not only is such a background of knowledge proper for a specialist in any branch of medicine, but it is also necessary for the full understanding of those conditions which are to be found only in the oral cavity.

Fortunately in Britain it is not difficult to provide such an educational background, for some of our dental schools are part of a medical college and teaching hospital. Where they are not an integral part there is usually a close association with a medical college or a teaching hospital group.

While it is easy to appreciate the need for such a course it is more difficult to define its objectives. There should be a brief general survey emphasizing the uniformity of approach to the problem of disease irrespective of the system affected. Such a survey helps to place dentistry in its proper perspective in relationship to the rest of medicine and also shows the student that the principles of diagnosis and treatment which he is being taught by his dental teachers are common to all the other specialties. Dental surgeons must be able to recognize the patient who is not fit enough for treatment, particularly if this involves minor surgery or a general anaesthetic. Cardiovascular, respiratory, and haematological disorders are important in this respect.

Many general medical conditions affect the oral tissues. The oral manifestations of these diseases are normally covered during the dental course, but it is important that the student hears about and sees something of the other aspects of these diseases or, for instance, every patient with a pigmented oral mucosa will be thought to be a case of Addison's disease.

The effects of modern drugs can require special precautions in the way the patient is treated. Indeed, the more successful the dental treatment of the patient and the longer he keeps a natural dentition, the more difficult may treatment become. Thus in many ways successful medical and dental treatments create their own problems.

There is much to do, but most university courses allow enough time for general subjects. With care an integrated course can be designed which groups together the appropriate lectures in pathology, microbiology, medicine, and surgery relating to a given topic. But who should teach the dental students? In days gone by the classes of dental students were small and there was a temptation to put them in with the much larger classes of medical students, or else to give them into the charge of a junior member of staff. Neither arrangement was satisfactory. Now that classes of 50 to 80 dental students are common the need to treat them separately is not questioned. While the factual content of the course is elementary its scope is large and it is difficult to teach well. Either a consultant who is an experienced teacher or a fulltime senior lecturer should be in charge of each subject. These teachers should have the dental students as their primary responsibility-and if their training in general medicine is to be viewed by dental students as an important part of their course the teaching must be enthusiastic. Equally important, medical students' knowledge of dentistry should be based on authoritative formal teaching.

\section{Sun-gazing}

By the end of the nineteenth century at least eight reports had described damage to the retina from looking at an eclipse of the sun. ${ }^{1}$ With the growth of medical publication the pace quickened in the present century, so that now virtually every solar eclipse has its sequel of documented casualties, the latest being a report of five cases in the B.M.7. a year ago. $^{2}$ Doubtless many more go unrecorded. Modern curiosity has replaced ancient terror at this heavenly concordance, and the result in the upshot may well be more injurious. The Ministry of Health has therefore very properly issued a warning to the people of Britain about the partial eclipse of the sun due on 22 September.

Damage to the retina from sun-gazing has also been recorded in cases of mental disturbance. Mr. M. J. Gilkes reports one in our correspondence columns this week at page 678. Scanning the sky for aircraft has been another cause of this injury, especially in wartime, as has staring towards the sun as an intended remedy to "strengthen the eyes" or in the course of religious devotions. ${ }^{3}$ All these are apt to cause a burnt-out hole in the retina, with perhaps oedema, minute haemorrhages, and some retinal detachment. According to Sir Stewart Duke-Elder ${ }^{1}$ the prognosis is extremely variable and to some extent doubtful owing to lack of follow-up for long periods, but he believes it will probably be good if the symptoms, particularly the scotoma, subside during the first month.

Socrates advised looking at a solar eclipse by watching its reflection in water, a procedure that is unsafe even in cloudy Britain and must have been riskier still in the clear skies of Greece. The Ministry of Health says the safe way

\footnotetext{
Duke-Elder, Sir S., Text-Book of Ophthalmology, Vol. 6, 1954 London.

2 Ridgway, A. E. A., Brit. med. F., 1967, 3, 212.

3 Das, T., Nirankari, M. S., and Chaddah, M. R., Amer. 7. Ophthal., 1956, 41, 1048.

4 Flynn, J. A. F., Med. 7. Aust., 1960, 1, 85.

- Gilkes, M. J., Osmond, A. H., Roberts, D. St. C., and Thorne Thorne, B., Brit. med. Ұ., 1961, 1, 424.

"Flynn, J. A. F., Brit. med. f., 1961. 1. 68.
} 
is to look through a filter of density 4.5 - "This means that a filter should not transmit more than one part in 30,000 of the total solar radiation." But who knows whether a filter, especially if improvised, has the right density ? In fact the best way for the amateur observer to see an eclipse is by projecting its image through a pin-hole on to a sheet of card or paper, ${ }^{45}$ which may be black ${ }^{1}$ or white. ${ }^{6}$

\section{Venous Ulcers}

The treatment of varicose veins and their complications is always a matter of controversy, and though it is a good sign that such a common condition ${ }^{1}$ can stimulate prolonged and heated activity in our correspondence columns it is also indicative of our ignorance of the basic abnormalities and that our treatment is often illogical and empirical. No one denies that the primary cause of venous (or varicose, or orthostatic-take your pick) ulcers is the venous hypertension which develops in incompetent veins during erect exercise, yet how this abnormality produces the induration, pigmentation, fat necrosis, eczema, and ulceration has not been satisfactorily explained. Numerous hypotheses have been advanced. The oedema has been blamed on changes of capillary permeability ${ }^{2}$ - a most difficult thing to measure in intact man. Changes in tissue nutrition have been blamed on the presence of arteriovenous fistulae, ${ }^{3-5}$ a suggestion that could also explain the increased oxygen tension of the blood draining the region of a venous ulcer. Capillary fragility with interstitial haemorrhage, fat necrosis, and ultimately ulceration may be due to either direct trauma from outside or indirect trauma from the internal activity of the muscles and the rapid changes of venous pressure. With so many possible aetiological factors it is not surprising that a multitude of remedies are in use, nor that the only ones that are really effective are those that control the venous hypertension-for this must be the prime cause. Thus the closure of the incompetent superficial-to-deep connecting veins by surgery ${ }^{6}$ or sclerotherapy ${ }^{7}$ will produce a permanent cure in most cases. Alternatively, simple elevation or lateral counter-pressure with elastic stockings, adhesive strapping, or impregnated bandages will produce a temporary cure, and such treatment is often necessary as an accompaniment or preliminary to the definitive treatment of the incompetent veins.

It is also a fair comment that any form of treatment aimed at the secondary effects of the venous hypertension is unlikely to be curative and may not help at all. Diuretics are of little value. Local or systemic antibiotics are not indicated, for most varicose ulcers are not infected with virulent pathogens -another interesting and unexplained feature. The medicaments in the impregnated bandages, such as zinc oxide and ichthyol, probably do no more than make the bandage stiffer and harder and consequently a better support. Interest has recently shifted towards the changes in capillary permeability, and substances such as troxerutin, which have been shown to affect capillary permeability, ${ }^{8}$ are claimed to be of value. The problems of assessing such drugs in the absence of an acceptable method of measuring capillary permeability in the margins of a venous ulcer in man are extremely difficult. Those studies so far published suggest that this type of approach will yield only small fringe benefits. ${ }^{9}$
The problem always comes back to the major known abnormality-venous hypertension-and unless this is abolished a permanent cure cannot be achieved. Yet this is more easily said than done and requires a painstaking clinical examination and repeated follow-up, for quite frequently the first assault misses some of the abnormal perforating vessels. The vast number of patients affected makes the use of special techniques such as phlebography or pressure studies impractical, and even these techniques are not infallible. So these patients must be treated on the basis of clinical examination, and because they may require more than one operation the relatively inexpensive injection techniques revived by G. W. Fegan ${ }^{7}$ are appealing. What matters most is that the whole problem of varicose veins and venous ulceration should be approached in an exact and scientific manner: the ability to produce rapid relief from the symptoms of this disease is of great economic importance.

\section{Cheltenham Meeting}

The Annual Clinical Meeting of the British Medical Association at Cheltenham next month is to be a joint one with the British Paediatric Association. The B.M.A.'s practice, which began last year, of inviting a specialist society to join in its clinical meetings is a good one. Since the division of the Annual Scientific Meeting into specialist sections was discontinued some seven years ago there has been a danger that the B.M.A. might be thought to have less concern for specialist medicine. The new practice is a valuable corrective to any such trend, and the Association's Board of Science, under the chairmanship of Professor Sir Ronald Tunbridge, may be expected to continue it.

The emphasis in the programme for the Cheltenham Meeting, printed in the Supplement, is on subjects of interest to the practising doctor. Among them are virus diseases, allergic reactions in the lungs, malabsorption syndromes, and a variety of special topics in paediatric medicine. The sessions are recognized for the purposes of the postgraduate training allowance for N.H.S. general practitioners. Lord Todd is to give the opening address entitled "The Doctor in the Changing World."

A more pleasant venue for a meeting than Regency Cheltenham, together with its beautiful surrounding country, could scarcely be imagined, and there is an attractive programme of visits to nearby places of interest. The Meeting coincides with the centenary of the Gloucestershire Branch of the B.M.A., and visitors will have the privilege of being able to join in a service of thanksgiving in Gloucester Cathedral on 27 October.

\footnotetext{
Borschberg, E., The Prevalence of Varicose Veins in the Lower Extremities, 1968. Basel.

2 Myers, T. T. in Peripheral Vascular Diseases, 1962, 3rd edition. ed. E. V. Allen, N. W. Barker, and E. A. Hines. London.

3 King, E. S. J., Aust. N.Z. \}. Surg., 1950, 20, 126.

- Piulachs, P., and Vidal-Barraquer, F., Angiology, 1953, 4, 59.

s Gius, J. A., Arch. Surg., 1960, 81, 299.

6 Dodd, H., and Cockett, F. B., The Pathology and Surgery of the Veins of the Lower Limb, 1956. London.

- Fegan, G. W., Varicose Veins, 1967. London.

- Wismer, R., Praxis, 1963, 52, 1412.

- Fitzgerald, D. E., Practitioner, 1967, 198, 406.
} 